\title{
Molecular Phylogenetic Identification of Plant-pathogenic Fungi Isolated from Two Medicinal Atractylodes macrocephala Koidz Species
}

\author{
Yun Huang (Corresponding author) \\ Department of Plant Pathology, Sichuan Agricultural University \\ Wen'jiang 611134, SiChuan Province, China \\ E-mail: 5787huangyun@sina.com \\ Hui Yang (Corresponding author) \\ Department of Plant Pathology, Sichuan Agricultural University \\ Wen'jiang 611134, SiChuan Province, China
}

Tel: 86-180-0804-5551 E-mail: yanghui981@sohu.com

Fan Liu

Department of Plant Pathology, Sichuan Agricultural University

Wen'jiang 611134, SiChuan Province, China

E-mail: sunshiny1983@yahoo.com.cn

Mengliang Tian

Department of Plant Pathology, Sichuan Agricultural University

Wen'jiang 611134, SiChuan Province, China

E-mail: secondat@sicau.edu.cn

Wenguang Li

Baoxing Science and Technology Bureau

Baoxing, Sichuan Province, China

E-mail: Lwqx1987@163.com

Received: January 10, 2012 Accepted: January 21, 2012 Online Published: May 16, 2012

doi: $10.5539 /$ jas.v4n6p290

URL: http://dx.doi.org/10.5539/jas.v4n6p290

\begin{abstract}
Plant-pathogenic fungi are a large and diverse group of organisms which exhibit great importance in agriculture and natural plant communities. In this study, we investigated the taxonomic identities and phylogenetic relationships of pathogenic fungi isolated from two medicinal Atractylodes macrocephala Koidz species, Swordlike A. macrocephala and Largehead A. macrocephala, using the morphological and molecular approaches. Morphological differences among the fungal isolates indicate that diverse distinct morphotypes might be present within the hosts. Forty fungal isolates were selected for further molecular phylogenetic analysis using the internal transcribed spaces (ITS1 and ITS2) of nuclear ribosomal DNA sequence and the intervening 5.8s gene region. While assessing diversity of Fusarium lateritium isolate and Gibberella zeae species from medicinal A. macrocephala using molecular approaches, Based on an 18S rRNA PCR approach, 4 fungal clone types were
\end{abstract}


detected in medicinal A.macrocephala, Our results suggest that F.lateritium isolate and G. zeae species are the dominant pathogenic fungi in the A.macrocephala hosts, and some of these pathogenic fungi exhibit host and tissue specificity at the phylogeny level, fungal clone type A11, B11, D11 belongs to G. zeae, while the other C11 clone type belongs to $F$. lateritium. This aspect can be further explored to understand the relationships between plant hosts and their fungal pathogenic. Moreover, we firstly found $F$. lateritium is the dominant pathogenic fungi in A. macrocephala.

Keywords: Atractylodes macrocephala Koidz, 18srRNA, Fungi, Taxonomy

\section{Introduction}

Plant-pathogenic fungi show great importance in agriculture and natural plant communities. These pathogenic fungi have evolved different lifestyles. The modes of plant-pathogen interaction also exhibit diversity. Some pathogens can synthesize and secrete toxic metabolites to kill the host cells at infection site and live off the dead tissue, which is referred to as necrotrophy. In contrast, biotrophic fungi do not produce toxins and they require living plants as a source of nutrients. Globally, there are at least one million species of plant-pathogenic fungi (Ganley and Newcombe, 2006). This represents an important genetic resource for biotechnology study. Plant-pathogenic fungi, especially the fungi colonizing medicinal plants, can produce secondary metabolites which have been recognized as potential sources for agricultural, pharmaceutical and industrial uses (Strobel and Daisy, 2003, Hyde and Soytong, 2008). In recent years, plant pathogenic fungi biodiversity, the bioactivity of pathogenic fungi metabolites and the host-pathogen relationship have been received more attention.

In Traditional Chinese Medicine (TCM), Bai Zhu (the rhizomes of Atractylodes macrocephala Koidz, a member of the Compositae) is widely distributed in the world, with about 185 species in China and is highly valued for its medicinal properties (LI et al., 2001). A.macrocephala can invigorate the spleen, and cure patients with anorexia, excessive perspiration, oedema, splenic asthenia, and abnormal fetal movement (Junhao et al., 1996, Hong et al., 2005). Several recent studies have shown that genetic methods can be successfully used in the studies of pathogenic fungi (Arnold and Lutzoni, 2007; Arnold, 2007; Poulsen et al., 2005; Wang et al., 2005; Morakotkarn et al, 2007; Huang et al, 2008); DNA-based techniques, particularly PCR can detect minute quantities of a pathogen. These methods have provided new opportunities to study and understand, for example, the biology of plant pathogenic fungi, pathogen population structure and dynamics, host- pathogen interactions, etc. These aims have been achieved by optimizing techniques (Mayayo et al., 1999). This mainly based on performing molecular targets, which serve as phylogenetic analysis (Yeo and Wong, 2002). One of targets is the group of genes which encode the nuclear ribosomal RNA (rRNA). Ribosomal DNA (rDNA) is the DNA sequences that direct the synthesis of ribosomal RNA. rDNA is considered a useful marker for phylo- genetic studies. The main reason is that its sequences encode multiple-copy loci. The repeated copies are synchronized by concerted evolution (Sugiyama et al., 1999). Furthermore, ribosome are present in all organisms (Van de Peer and De Wachter, 1997), Parts of the molecule are conserved. Therefore, they may serve as reference for evolutionary divergence studies. The $5.8 \mathrm{~S}, 18 \mathrm{~S}$, and $25-28 \mathrm{~S}$ rRNA genes (or rDNAs) are generated from a $35 \mathrm{~S}$ to $40 \mathrm{~S}$ precursors, which are spliced out of the transcript. The sequencing of rDNA and internal transcribed spacers (ITS) was applied to fungal taxonomy. Recently, the accumulation of sterile isolates obtained in entophyte surveys obviously improves taxonomic information (Duong et al., 2006; Promputtha et al., 2005; Higgins et al., 2007; Crozier et al., 2006).

\section{Materials and Methods}

\subsection{Plant Material}

Healthy plants of medicinal A. macrocephala growing in Baoxin was sampled from October 2010 to June 2011. A typical symptom of diseased A. macrocephala plants were collected from Baoxin, West Sichuan China. Fresh samples were taken to the laboratory and treated within 8 hours. Digital photographs were taken of each sample, and all the samples were deposited in School of Biological Sciences, Research Center for Pathogenic fungi, Sichuan Agricultural University.

\subsection{Isolation of Pathogenic Fungi}

A total of 30 samples of both roots and stems from the A. macrocephala were first washed in running water. The roots and stems were cut into pieces $(5 \mathrm{~mm}$ in length). Surface sterilization and isolation of Pathogenic fungi followed a modified procedure as described by Schulz et al. (1993), and the details of the procedure were also given in Huang et al. (2007). 


\subsection{DNA Extraction, Amplification and Sequencing of Fungal ITS}

A total of 2 representative isolates of various morphological Pathogenic fungi isolated from each $A$. macrocephala species were selected for molecular identification. Fungal genomic DNA was prepared with the NucleoSpin ${ }^{\circledR}$ DNA extraction Kit (Macherey- Nagel, Düren, Germany). The fungal mycelia grew on PDA for 5-10 days at $25^{\circ} \mathrm{C}$ and were suspended in $400 \mu \mathrm{l}$ of buffer $\mathrm{C} 1$. About $10 \mathrm{mg}$ of fungal mycelia were scraped from fresh culture with a sterile nipper. All the extraction procedure accorded to the instructions which was given in the kit user's manual. The extracted DNA pellet was kept at $-20^{\circ} \mathrm{C}$.PCR reactions were carried out in a PTC$100^{\mathrm{TM}}$ thermal cycler (MJ Research, Inc., Watertown, MA, USA) with universal primers ITS1 (5'-TCCGTAGGTGAACCTGCGG-3') and ITS4 (5'-TCCTCCGCTTATTGATATGC-3'). Amplified fragment includes ITS1, 5.8S and the ITS2 of rDNA. Amplification was performed in a $25 \mu 1$ reaction mixture containing $2 \mathrm{x}$ Taq PCR Master Mix $12.5 \mu \mathrm{L}, 2 \mu \mathrm{l}$ primes IST1-F and ITS4-R primers, $3 \mu 1$ of fungal genomic DNA and mini-pure water $7.5 \mu \mathrm{L}$. The PCR product was purified with High Pure PCR Product Purification Kit (Roche Diagnostics GmbH, Germany). Direct DNA sequencing was performed using primers ITS1 (5'-GCATCGATGAAGAACGCAGC-3') and ITS5 on an ABI 3100 automated sequencer following the manufacturer's instructions (Applied Biosystems, Inc.).

\subsection{DNA Sequence Assembly and Alignment}

Sequence similarity searches were performed for each of the 30 representative fungal sequences against the non-redundant database maintained by the National Center for Biotechnology Information using the BLAST algorithm (http://www.ncbi.nlm.nih.gov). The ITS1-5.8S-ITS2 sequences of pathogenic fungi isolates were aligned with the sequences of selected reference taxa using the ClustalW and the alignment was inspected and adjusted manually where necessary. The nucleotide sequences of reference taxa along with their GenBank accession numbers are listed in Table 2. GenBank accession numbers of the 10 representative pathogenic fungi sequences from this study and their top BLAST match sequences are given in Table 3.

\subsection{Phylogenetic Analysis}

Phylogenetic analysis was conducted based on both the ITS and 5.8S gene data using both maximum parsimony (MP) and neighbor joining (NJ) approaches. MP and NJ searches were carried out using MEGA*4.0.2 All characters were equally weighted and unordered. Alignment gaps were treated as missing data. MP analysis was conducted using a heuristic search with treebisection-reconnection (TBR) branch swapping and 100 random addition sequence replicates. Statistical support for the internal branches was estimated by bootstrap analysis based on 5,000 replications. NJ trees were constructed based on the total character differences and bootstrap values were calculated from 1,000 replications.

\section{Results}

\subsection{Isolation and Morphological Grouping of Endophytic Fungi from Two Medicinal A. macrocephala}

Morphological identification of the 40 fungal isolates from two medicinal A. macrocephala was first carried out according to colony or hyphal morphology of the fungal culture, characteristics of the spores, and reproductive structures if discernible (Barnett et al., 1998). Based on these features, the 36 pathogenic fungi could be classified into 2 different morphological taxa (Table 1). Most of the fungal isolates (25 isolates) were obtained from host Swordlike A. macrocephala, 14 isolate isolated from its root, 9 isolates from stems. 15 fungal isolates were isolated from host Largehead A. macrocephala, 8 from its roots and 5 from stems. Using the traditional morphological techniques, only some of the fungal isolate could be identified to the genus level. Among them, whilst cultural techniques are unsuitable for detection of fungi that are slow growing, or non-culturable in virto and the choice of the medium may select the species recovered and some fungal isolates $(10 \%)$ lacking sporulating structures failed to speculate.

\subsection{Molecular Identification and Phylogenetic Analysis of Representative Pathogenic Fungi}

In addition to the morphological characterization, molecular analyses were carried out to confirm the identification of 2 representative fungal isolates from the two medicinal A. macrocephala. Sequence analysis of the ITS consensus sequences (509-535 bp) of the isolate pathogenic fungi were identified in Figure 1.

The sequences from fungal clone types A11, B11, D11 and C11 were further examined through BLAST and FASTA searches and through direct comparisons with potentially related taxa. The highest match was to a $F$. lateritium isolate HMA-1 sequence with the next closest matches to F. lateritium (AF310980.1) and F.tricinctum (AB470878.1) species. These 2 genera are possibly closely related members of the Fusarium, and so the clone type $\mathrm{C} 11$ fragment was aligned against the 5 best matches from that order, some further representative species of these genera and representative sequences of the other related fungal orders that were included in the 10 best 
matches. The 10 protein-coding genes for each of the fungi were considered as a single unit that was used to construct neighbor-joining (NJ) and maximum parsimony (MP) phylogenetic tree and Ustilaginoidea virens (AB092953.1) was used as an outgroup accepting the fact that amino acid sequences are more informative for analyzing sequences as divergent as the refrence genes (Bullerwell et al., 2003, Gray et al., 1999) (Fig. 2 and Fig. 3). A phylogenetic tree (Fig. 2) recovered clone type $\mathrm{C} 11$ and the 1 best matches in a distinct group within a larger F. lateritium, and some of these were also represented by marine genera. These were linked to, but distinct from, a group formed largely of species that were pathogens of other fungi. So F.lateritium is the dominant Pathogenic fungi firstly detected in A. macrocephala. And the $F$. lateritium grouping was well supported by a terminal bootstrap value of $100 \%$. The distance-based phylogenetic reconstruction also supported this grouping based on the small evolutionary distances within the $F$. lateritium Isolates within this group had differences ranging from $0 \%$ to $3 \%$ and evolutionary distance less than $3.8 \%$ (Table 2, Table 3 ).

Sequences from clone types A11, B11 and D11 showed $98 \%$ similarity over $100 \%$ of the sequence to a range of fungal genera that are all placed in the G.avenacea. In addition, clone A11, B11 and D11 showed 100\% similarity over $94 \%$ of the sequence to 1 sequence from $F$. culmorum (AY147313.1). The sequences were included in a phylogenetic analysis with similar sequences from a range of G.avenacea species (Fig. 3).Clone types clone A11, B11 and D11 were recovered together with the G. zeae (JF303858.1) sequence in a distinct clade. Bootstrap confidence levels were high for these groupings, but generally low across this dataset.

\section{Discussion}

In this study, we examined the genetic diversity of $F$. spp. and G. zeae Spp. isolates derived from medicinal $A$. macrocephala in Research Center for Pathogenic fungi, Sichuan Agricultural University. Toward this end, we studied the feasibility of using the ITS region for the detection and identification of $F$. spp. and G. zeae spp. to the species level. In addition, we compared this molecular classification technique to classification based on examination of morphologic characteristics.

\subsection{Diversity of Plant-pathogenic Fungal in This Study}

In this study, 40 Plant-pathogenic fungal isolates were obtained from Swordlike A. macrocephala and Largehead A. macrocephala, for fungal identification, we relied on a combination of traditional and molecular methods. Four of the representative morphological isolates were further identified with molecular phylo-genetic analysis of ITS1-5.8S-ITS2 sequences. Generally, there was a good agreement between morphological and ITS-sequence based approaches. The Plant-pathogenic fungal communities associated with the two A.macrocephala species showed high species diversity. In addition, fungal species compositions were distinct in different tissues. These fungals could be identified to species, genus, or family level, mainly including F. lateritium and G. zeae. G. zeae was the most frequent Plant-pathogenic fungal either within or among the hosts, consistent with the findings reported in other studies of G.zeae (Yun et al., 2000, Wildermuth et al., 1997). In this study, two Plant-pathogenic fungal isolates of morphological were isolated from stems and roots of A.macrocephala. G. zeae is common Plant-pathogenic fungal in many species(Lee et al., 2009, Pereyra and Dill-Macky, 2008) and we reported the phylogenetic significance of morphological character in the taxonomy of G. zeae species. However, F. lateritium is the dominant Pathogenic fungi firstly detected in A. macrocephala.

\subsection{Taxonomy and Phylogeny of Plant-pathogenic Fungal Isolates in This Study}

The major polygenetic relationships were congruent based on MP, NJ, and except for minor differences in the placement of some small clades, which had not affected fungal strain identification.

All the 36 Plant-pathogenic fungal were obtained from host $A$. macrocephala, which accounted for $90 \%$ of its total fungal isolates. Of the four isolates, A11, B11, C11 and D11 were selected for molecular identification; only one or two nucleotides were different in their ITS1-5.8S-ITS2 sequences. A11, B11 and D11 were similar in morphological characteristics, and the three isolates from the stems, Sequences alignment showed that they were $100 \%$ identical to many closely related species of G. spp, with G. zeae as the most frequently matched species. In the present study, G. zeae isolates obtained from roots and stems idetical in their ITS1-5.8S-ITS2 sequences, suggesting that they may belong to the same fungal taxa. As indicated by their sequence homology to the corresponding GenBank accessions, the two isolates from stems (A11 and D11) and the one from roots (B11) might belong to G. zeae, This study showed that G. zeae species are the most frequent Plant-pathogenic fungal in A.macrocephala. In our phylogenetic analysis, most of the three representative fungal isolates could be identified as G. zeae, which were sexual phases of Gibberella. However, clone type $\mathrm{C} 11$ and the 1 best match in a distinct group within a larger F.lateritium, and some of these were also represented by marine genera. These were linked to, but distinct from, a group formed largely of species that were pathogens of other fungi. So F. lateritium is the dominant Pathogenic fungi firstly detected in A. macrocephala in our research. 


\subsection{The Use of Molecular Technique in Identifying Plant-pathogenic Fungal}

Traditionally, microscopic and cultural techniques are often available for detecting and enumerating fungal spores. However, the methods are time-consuming and laborious. They require skilled and highly specialized expertise. Furthermore, microscopy is often fallible for identification of non-descript spores. Endophytic fungi are assessed by rolling the suface-sterilized plant tissue samples, and subsequently isolation of fungal endophytes which grow in samples place onto culture media (Bills et al., 1996, Devarajan and Suryanarayanan, 2006). However, cultural techniques are inappropriate for detection of slow growing or non-culturable fungi in vitro (Lacap et al., 2003). Fungi that emerge from these samples can be identified by means of phenotypic (morphological) or genotypic (molecular) characters. In contrast, molecular techniques are sensitive and specific for indentifying microorganisms. They can be used for the taxonomic classification and identification of microbial strains (Sette et al., 2006). The internal transcribed spacer (ITS) has been used innumerous systematic studies at genus and species levels of a wide array of plant taxa (Alice and Campbell, 1999, Sang et al., 1995). ITS-1 and ITS-2 are two internal spacers which locate between genes encoding the 18, 5.8 and 26s nuclear ribosomal RNA (nrRNA) subunits. In addition, the 5.8s nrRNA are referred as nrDNA ITS region (Baldwin, 1992). The length of ITS- 1 and ITS- 2 are less than $300 \mathrm{bp}$. The length of $5.8 \mathrm{~s}$ subunit is almost invariant. It is around $160 \mathrm{bp}$ within angiosperms. This makes the entire ITS region less than $700 \mathrm{bp}$. Therefore, this is easy for sequencing and analysis. ITS region belongs to the nrDNA family, which undergo rapid concerted evolution. Its rapid concerted evolution, small size and ease of amplification made nrDNA ITS region as a phylogenetic standpoint and also promote accurate reconstruction of species relationship. Many studies revealed a phylogenetic relation among different species (Baldwin et al., 1995). Based on DNA analysis, problems associated with taxonomic identification of mycelia sterilia could be solved. However, molecular analysis alone also has limitations. It cannot overcome the problem of over-isolating fast growing fungal species at the expense of slow growing taxa, nor isolating species that will not grow in culture (Duong et al., 2006, Hyde and Soytong, 2008). The use of ITS sequences also has limitations in phylogenetic analysis. Because the noncoding ITS sequence is fast evolving with many variable characters, it is usually difficult to achieve a perfect sequence alignment at high taxonomic levels. Moreover, it has been shown that $20-30 \%$ of sequences downloaded from GenBank for comparative analysis may not be accurate in their identification (Nilsson et al., 2006, Hyde and Soytong, 2008)). This inaccuracy in the database may have contributed to some of the unexpected observations in our study, such as F. lateritium and G. zeae all having their accessions scattered across the phylogenetic tree, whereas some different species (e.g., species of F. culmorum and G. zeae) being clustered within the same clade. Further studies using different gene sequences can be conducted to resolve this type of difficulties in the phylogenetic analysis of fungi.

\section{Conclusion}

In summary, the ITS region provides a sufficient genetic scaffolding to detect and reliably differentiate $F$. spp. and G.zeae spp. isolated in medicinal A.macrocephala. In recent years, DNA-based technology is widely used. We can detect and classify the isolated fungal precisely and rapidly using the DNA-based technology. The nrDNA ITS sequence has already been proved the usefulness in phylogenic analysis of F.lateritium isolates and G.zeae species according to the present study. These studies also provide substantial basis for future phylogenic and evolutionary analysis among the groups belongs to the family $F$. spp. and G. zeae spp.. Our results will be helpful to furtherly study the biogeographical and molecular evolutionary, their phylogenic origins and intraspecies population.

\section{References}

Alice, L. A., \& Campbell, C. S. (1999). Phylogeny of Rubus (Rosaceae) based on nuclear ribosomal DNA internal transcribed spacer region sequences. American Journal of Botany, 86, 81.

Arnold, A. E. (2007). Understanding the diversity of foliar endophytic fungi: progress, challenges, and frontiers. Fungal Biology Reviews, 21, 51-66.

Arnold, A. E., \& Lutzoni, F. (2007). Diversity and host range of foliar fungal endophytes: Are tropical leaves biodiversity hotspots? Ecology, 88, 541-549.

Baldwin, B. G. (1992). Phylogenetic utility of the internal transcribed spacers of nuclear ribosomal DNA in plants: An example from the compositae. Molecular Phylogenetics and Evolution, 1, 3-16.

Baldwin, B. G., Sanderson, M. J., Porter, J. M., Wojciechowski, M. F., Campbell, C. S., \& Donoghue, M. J. (1995). The ITS region of nuclear ribosomal DNA: a valuable source of evidence on angiosperm phylogeny. Annals of the Missouri Botanical Garden, 247-277.

Barnett, P., Hemrika, W., Dekker, H. L., Muijsers, A. O., Renirie, R., \& Wever, R. (1998). Isolation, 
characterization, and primary structure of the vanadium chloroperoxidase from the fungus Embellisia didymospora. Journal of Biological Chemistry, 273, 23381. http://dx.doi.org/10.1074/jbc.273.36.23381

Bills, G. F., Redlin, S., \& Carris, L. (1996). Isolation and analysis of endophytic fungal communities from woody plants. Endophytic fungi in grasses and woody plants: systematics, ecology, and evolution, 31-65.

Bullerwell, C., Forget, L., \& Lang, B. (2003). Evolution of monoblepharidalean fungi based on complete mitochondrial genome sequences. Nucleic Acids Research, 31, 1614. http://dx.doi:10.1093/nar/gkg264

Crozier, J., Thomas, S., Aime, M., Evans, H., \& Holmes, K. (2006). Molecular characterization of fungal endophytic morphospecies isolated from stems and pods of Theobroma cacao. Plant pathology, 55, 783-791. http://dx.doi.org/10.1016/j.mycres.2008.01.008

Devarajan, P., \& Suryanarayanan, T. (2006). Evidence for the role of phytophagous insects in dispersal of non-grass fungal endophytes. Fungal diversity, 23, 111-119.

Duong, L. M., Jeewon, R., Lumyong, S., \& Hyde, K. D. (2006). DGGE coupled with ribosomal DNA gene phylogenies reveal uncharacterized fungal phylotypes. Fungal Divers, 23, 121-138.

Ganley, R. J., \& Newcombe, G. (2006). Fungal endophytes in seeds and needles of Pinus monticola. Mycological Research, 110, 318-327. http://dx.doi.org/10.1016/j.mycres.2005.10.005

Gray, M. W., Burger, G., \& Lang, B. F. (1999). Mitochondrial evolution. Science, 283, 1476.

Higgins, K. L., Arnold, A. E., Miadlikowska, J., Sarvate, S. D., \& Lutzoni, F. (2007) Phylogenetic relationships, host affinity, and geographic structure of boreal and arctic endophytes from three major plant lineages. Molecular Phylogenetics and Evolution, 42, 543-555. http://dx. doi:10.1016/j.ympev.2006.07.012

Hong, S. H., Jeong, H. J., Chung, H. S., Kim, H. R., Chae, H. J., Shin, T., Seo, Y., \& Kim, H. M. (2005). An herbal formula, Herbkines, enhances cytokines production from immune cells. Journal of ethnopharmacology, 98, 149-155. http://dx.doi.org/10.1016/j.jep.2005.01.003

Huang, W., Cai, Y., Hyde, K., Corke, H., \& Sun, M. (2008). Biodiversity of endophytic fungi associated with 29 traditional Chinese medicinal plants. Fungal diversity, 33, 61-75. Hyde, K., \& Soytong, K. (2008) The fungal endophyte dilemma. Fungal Divers, 33, 163-173. http://dx.doi.org/10.1631/jzus.B0860005

Ichinoe, M., Kurata, H., Sugiura, Y., \& Ueno, Y. (1983). Chemotaxonomy of Gibberella zeae with special reference to production of trichothecenes and zearalenone. Applied and environmental microbiology, 46, 1364.

Junhao, M., Zhiliang, L., Qunli, Z., \& Hong, R. (1996). Effects of the polysaccharide of Atractylis macrocephala Koidz on the function of mouse T lymphocyte. Immunological Journal, 4.

Lacap, D., Hyde, K., \& Liew, E. (2003). An evaluation of the fungal 'morphotype'concept based on ribosomal DNA sequences. Fungal diversity, 12, 53-66.

Lee, J., Chang, I. Y., Kim, H., Yun, S. H., Leslie, J. F., \& Lee, Y. W. (2009). Genetic diversity and fitness of Fusarium graminearum populations from rice in Korea. Applied and environmental microbiology, 75, 3289. http://dx.doi.org/10.1128/AEM.02287-08

LI, W., WEN, H., ZHANG, A., GE, J., \& WU, H. (2001). Study on Quality of Atractylodes macrocephala Koidz. I-Determination of 2 Atractylenolides by HPLC [J]. Chinese Journal of Pharmaceutical Analysis, 3.

Mayayo, E., Pujol, I., \& Guarro, J. (1999). Experimental pathogenicity of four opportunist Fusarium species in a murine model. Journal of medical microbiology, 48, 363. http://dx.doi.org/10.1099/00222615-48-4-363

Morakotkarn, D., Kawasaki, H., \& Seki, T. (2007). Molecular diversity of bamboo-associated fungi isolated from Japan. FEMS microbiology letters, 266, 10-19. http://dx.doi.org/10.1111/j.1574-6968.2006.00489.x

Nilsson, R. H., Ryberg, M., Kristiansson, E., Abarenkov, K., Larsson, K. H., \& K ljalg, U. (2006). Taxonomic reliability of DNA sequences in public sequence databases: a fungal perspective. PLoS One, 1, e59.

Pereyra, S., \& Dill-Macky, R. (2008). Colonization of the residues of diverse plant species by Gibberella zeae and their contribution to Fusarium head blight inoculum. Plant Disease, 92, 800-807.

Poulsen, K. H., Nagy, R., Gao, L. L., Smith, S. E., Bucher, M., Smith, F. A., \& Jakobsen, I. (2005). Physiological and molecular evidence for Pi uptake via the symbiotic pathway in a reduced mycorrhizal colonization mutant in tomato associated with a compatible fungus. New Phytologist, 168, 445-454.

Promputtha, I., Jeewon, R., Lumyong, S., McKenzie, E., \& Hyde, K. (2005). Ribosomal DNA fingerprinting in the identification of non sporulating endophytes from Magnolia liliifera (Magnoliaceae). Fungal diversity, 20, 
167-186. http://dx.doi.org/10.1007/s00248-006-9117-x

Sang, T., Crawford, D. J., Stuessy, T. F., \& Silva, M. (1995). ITS sequences and the phylogeny of the genus Robinsonia (Asteraceae). Systematic Botany, 55-64.

Sette, L., Passarini, M. R. Z., Delarmelina, C., Salati, F., \& Duarte, M. C. T. (2006). Molecular characterization and antimicrobial activity of endophytic fungi from coffee plants. World Journal of Microbiology and Biotechnology, 22, 1185-1195.

Strobel, G., \& Daisy, B. (2003). Bioprospecting for microbial endophytes and their natural products. Microbiology and Molecular Biology Reviews, 67, 491. http://dx.doi.org/10.1128/MMBR.67.4.491-502.2003

Sugiyama, M., Ohara, A., \& Mikawa, T. (1999). Molecular phylogeny of onygenalean fungi based on small subunit ribosomal DNA (SSU rDNA) sequences. Mycoscience, 40, 251-258.

Van de Peer, Y., \& De Wachter, R. (1997). Evolutionary relationships among the eukaryotic crown taxa taking into account site-to-site rate variation in $18 \mathrm{~S}$ rRNA. Journal of molecular evolution, 45, 619-630. http://dx.doi.org/ 10.1007/PL00006266

Wang, S. Y., Chen, P. F., \& Chang, S. T. (2005). Antifungal activities of essential oils and their constituents from indigenous cinnamon (Cinnamomum osmophloeum) leaves against wood decay fungi. Bioresource technology, 96, 813-818. http://dx.doi.org/10.1016/j.biortech.2004.07.010

Wildermuth, G., Thompson, J., Robertson, L., Clarke, A., \& Wylie, P. (1997). Biological change: diseases, insects and beneficial organisms. Sustainable crop production in the sub-tropics: an Australian perspective, 112-130.

Yeo, S. F., \& Wong, B. (2002). Current status of nonculture methods for diagnosis of invasive fungal infections. Clinical microbiology reviews, 15, 465. http://dx.doi.org/10.1128/CMR.15.3.465-484.2002

Yun, S. H., Arie, T., Kaneko, I., Yoder, O., \& Turgeon, B. G. (2000). Molecular organization of mating type loci in heterothallic, homothallic, and asexual Gibberella/Fusarium species. Fungal Genetics and Biology, 31, 7-20. http://dx.doi.org/10.1006/fgbi.2000.1226.

Table 1. Number and morphological taxonomic identification of the Pathogenic fungi isolated from two A.macrocephala species

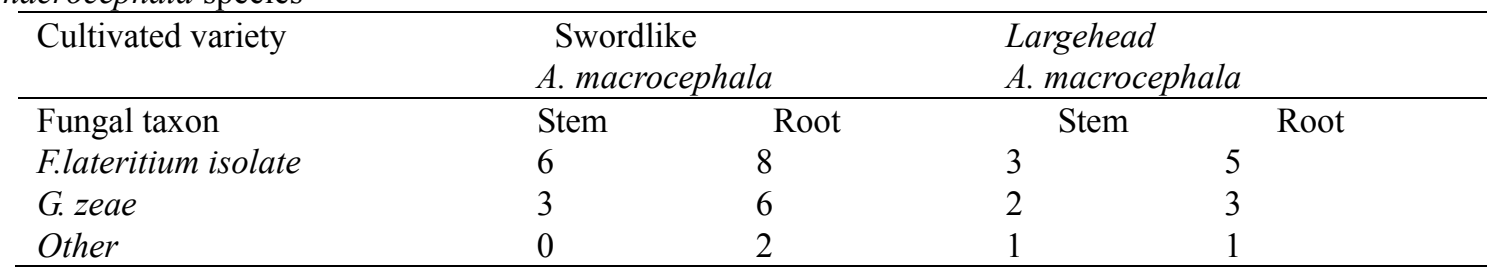

Table 2. Morphological identification, GenBank accession numbers and top BLAST match sequences of the fungal isolates from $A$. macrocephala included in phylogenetic analysis

\begin{tabular}{llllll}
\hline Fungal strain & $\begin{array}{l}\text { Morphological } \\
\text { identification }\end{array}$ & $\begin{array}{l}\text { GeneBank } \\
\text { accession } \\
\text { No. }\end{array}$ & Reference accession No. & coverage & $\begin{array}{l}\text { Max } \\
\text { ident }\end{array}$ \\
\hline A11,B11,D11 & G.zeae & EU255799.1 & G.avenacea isolate FA09 & $97 \%$ & $99 \%$ \\
C11 & F. lateritium isolate & GU480949.1 & $\begin{array}{l}\text { F.lateritium isolate } \\
\text { HMA-1 }\end{array}$ & $97 \%$ & $99 \%$ \\
\hline
\end{tabular}

Table 3. Species and GenBank accession number used in the study

\begin{tabular}{llll}
\hline Species & GeneBank accession No & Species & GeneBank accession No \\
\hline G.avenacea & HQ020475.1 & G. zeae & HQ176433.1 \\
G. avenacea & HQ020473.1 & G.zeae & GQ466391.1 \\
F. tricinctum & AB369452.1 & G. zeae & HQ176432.1 \\
F. lateritium & GU480949.1 & G. zeae & GU327636.1 \\
F. lateritium & AF310980.1 & G. zeae & JF303858.1 \\
G. avenacea & EU255803.1 & G. zeae & EU255799.1 \\
F. tricinctum & AB470878.1 & F. sp. & FJ614630.1 \\
F.sp. ZL-GT & HQ832677.1 & F. sp. & HQ630964.1 \\
F. oxysporum & AB470850.1 & F. culmorum & AY147313.1 \\
U. virens $\mathrm{U} 16$ & AB092953.1 & U. virens U16 & AB092953.1 \\
\hline
\end{tabular}




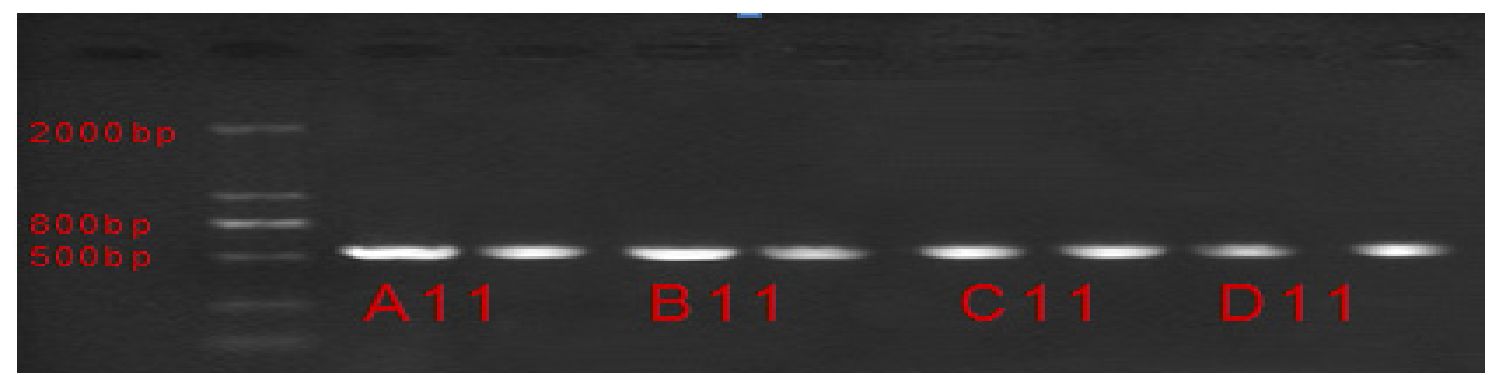

Figure 1. nrDNA ITS region amplified from A. macrocephala using IST1-F and ITS4-R primers (Biogene, USA)

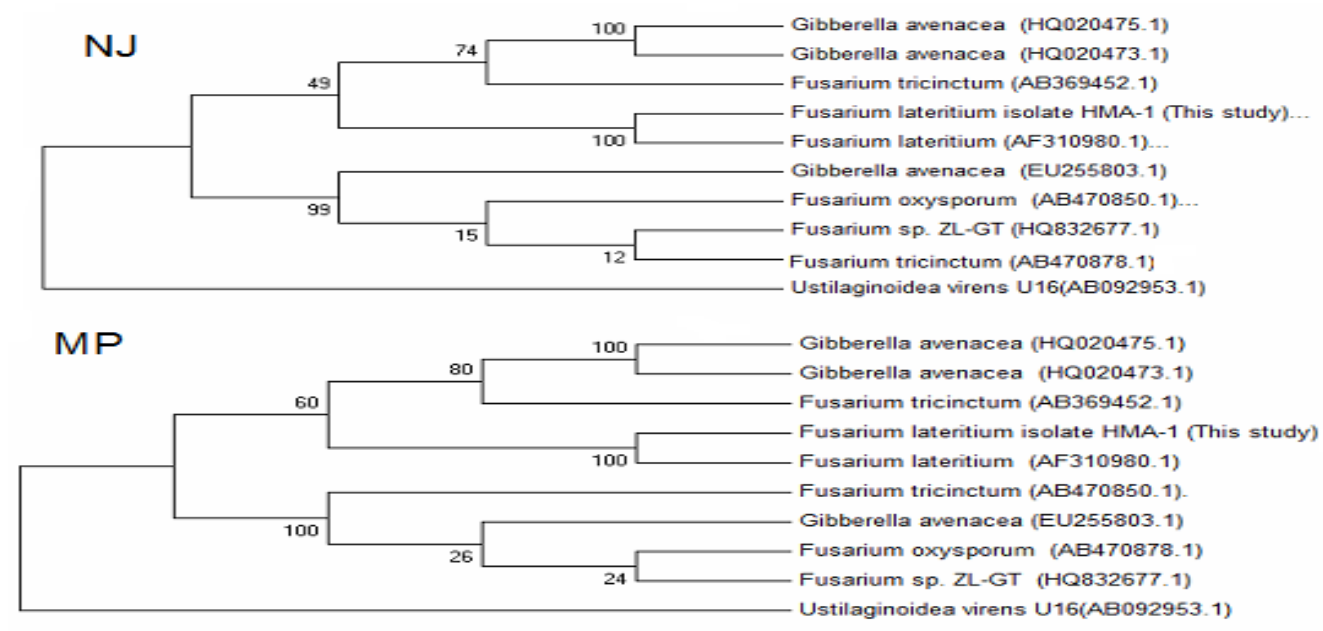

Figure 2. Maximum likelihood tree showing the relationship between fungal clone sequence type $\mathrm{C} 11$ and available sequences from databases

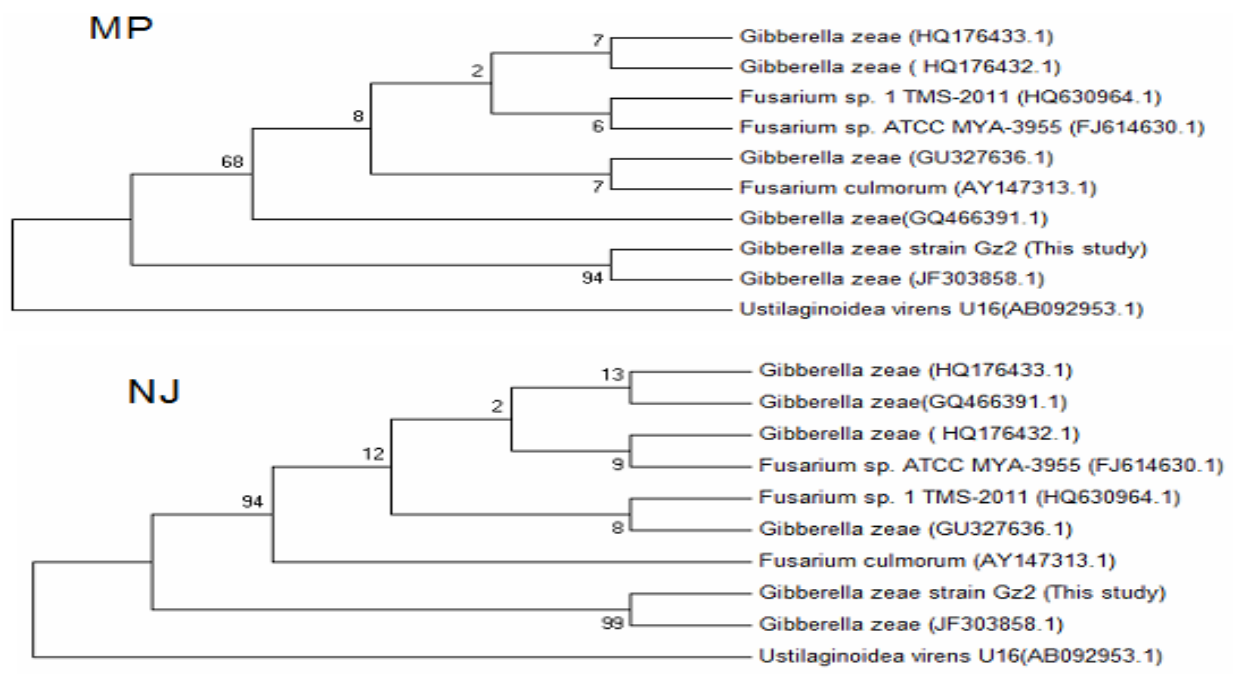

Figure 3. Maximum likelihood tree showing the relationship between fungal clone sequence types A11, B11 and D11 and available sequences from databases 